\title{
Comparação do ciclo alongamento-encurtamento em saltos verticais entre mulheres ativas adultas-jovens e idosas
}

\section{Comparison of cycle stretching-stretching in vertical jumps between adult and older active women}

\author{
Hyago Bernardes da Rosa', Igor Martins Barbosa², Eduardo Porto Scisleskiª, \\ Samuel Klippel Prusch4, Luiz Fernando Cuozzo Lemos 5 \\ Graduando do curso de Educação Física do Centro Universitário Cenecista de Osório (UNICNEC). Osório, RS, Brasil. <hyagobrosa@gmail.com> \\ 2 Graduando do curso de Educação Física do UNICNEC (Coordenação). Osório, RS, Brasil. <igormartinsbarbosa2@gmail.com> \\ Graduando do curso de Educação Física do UNICNEC. Osório, RS, Brasil. <dudupspb@hotmail.com> \\ ${ }^{4}$ Graduando do curso de Educação Física do UNICNEC. Osório, RS, Brasil. < samuel klippel@yahoo.com.br> \\ ${ }^{5}$ Doutor em Ciências do Movimento Humano pela Universidade Federal do Rio Grand̄e do Sul (UFRGS). Osório, RS, Brasil. <luizcanoagem@yahoo.com.br>
}

\section{ARTICLE INFO}

\section{Article history}

Received: 09/03/2018

Accepted: 10/07/2018

\section{Correspondent Author}

Igor Martins Barbosa

Rua: Vinte e Quatro de Maio, 141

95520-000 Osório, RS, Brasil

<igormartinsbarbosa2@gmail.com>

(C) 2018 All rights reserved

\section{Editors}

Alfredo Cataldo Neto

Paula Engroff

\begin{abstract}
RESUMO
OBJETIVO: Verificar se há economia eletromiográfica e/ou aumento nas varáveis dos saltos verticais, ambos em função da utilização do Ciclo Alongamento-Encurtamento e, comparar os achados entre mulheres jovens e idosas, ambas ativas. MÉTODOS: A amostra foi constituída por um grupo de idosos (GI) e um grupo de jovens (GJ), ambos do sexo feminino. Foi mensurada a potência através da plataforma de contato e atividade eletromiográfica dos músculos: bíceps femoral (BF), reto femoral (RF), gastrocnêmio medial e vasto lateral, através de testes de saltos verticais: Squat Jump (SJ) e Counter Movement Jump (CMJ). RESULTADOS: Todos os músculos avaliados, não apresentaram diferença estatisticamente significativa, na ativação eletromiográfica e nas comparações do percentual médio de utilização do Ciclo Alongamento-Encurtamento (CAE), mas se nota que para os músculos vasto lateral e reto femoral houveram diferenças significativas nas comparações entre os dois tipos de saltos de ambos os grupos, GI $(236,89 \pm 115,66$ para o SJ e 230,45 $\pm 109,10$ para

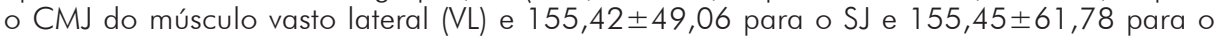
CMJ do músculo RF necessitou de maior ativação neuromuscular para saltar menos que o $\mathrm{GJ}$ $(117,40 \pm 133,09$ para o SJ e 133,09 $\pm 60,71$ para o CMJ do músculo VL e 106,72 $\pm 34,15$ para o SJ e 108,87 $\pm 38,85$ para o CMJ do músculo RF). CONCLUSÕES: O presente estudo constata que não houve diferença estatisticamente significativa na utilização do $C A E$, em ambos os grupos (Gl e GJ). Porém, quando se trata da altura saltada e a potência desenvolvida, houveram diferenças estatisticamente significativas na comparação dos grupos.
\end{abstract}

DESCRITORES: Ciclo Alongamento-Encurtamento; biomecânica; eletromiografia.

\begin{abstract}
PURPOSE: To verify if there is an electromyographic economy and/or an increase in the variables of vertical jumps, both as a function of the use of the Stretch-Shortening Cycle and to compare the findings between young and old women, both active. METHODS: The sample consisted of a group of elderly (GI) and a group of young (GJ), both female. The power was measured through the contact platform and electromyographic activity of the muscles: biceps femoris (BF), rectus femoris (RF), medial gastrocnemius and vastus lateralis, through vertical jumping tests: Squat Jump (SJ) and Counter Movement Jump (CMJ). RESULTS: All the muscles evaluated did not present a statistically significant difference in the electromyographic activation and the comparisons of the mean percentage of use of the Stretch-Shortening Cycle (CAE), but it was observed that for the vastus lateralis and rectus femoris muscles there were significant differences in the comparisons between the two types of jumps of both groups, GI (236.89 \pm 115.66 for SJ and $230.45 \pm 109.10$ for vastus lateralis muscle (VL) CMJ and 155.42 \pm 49.06 for SJ and 155, $45 \pm 61.78$ for the CMJ of the RF muscle) required greater neuromuscular activation to jump less than GJ (1 17.40 133.09 for SJ and 133.09 \pm 60.71 for VL muscle CMJ and 106.72 \pm 34.15 for the SJ and $108.87 \pm 38.85$ for the CMJ of the RF muscle). CONCLUSIONS: The present study confirms that there was no statistically significant difference in the use of CAE in both groups (GI and GJ). However, when it comes to the skipped height and the power developed, there were statistically significant differences in the comparison of the groups. KEYwORDS: Stretching-Shortening Cycle; biomechanics; electromyography.
\end{abstract}




\section{INTRODUÇÃO}

$\mathrm{Na}$ atualidade o número de pessoas idosas aumentou consideravelmente, passando esse público a ser alvo de pesquisas científicas, havendo preocupação e atenção por parte dos governantes, a fim de reduzir gastos com a saúde pública. Pimentel et al. ${ }^{1}$, afirmam que o processo de envelhecimento normalmente se configura por uma diminuição das funções fisiológicas entre os 30 e 70 anos e, a partir dessa faixa etária tais reduções sofrem ampliação da magnitude de declínio. Somado a isso, as funções motoras são fortemente comprometidas nesses indivíduos. Tal fato ocorre, pois há diminuição da massa muscular, provavelmente medida pela redução do número e no tamanho das fibras musculares individuais (em especial as fibras de contração rápida) ${ }^{2}$ e, assim a velocidade de condução nervosa pode diminuir ${ }^{3}$. Diante as referidas perdas, os indivíduos dessa faixa etária podem sofrer quedas em função do enfraquecimento muscular. Esses acontecimentos podem ser causados, por exemplo, pela diminuição da capacidade dos músculos extensores de joelho e quadril em desenvolver força rapidamente, e desta forma, podem prejudicar o desempenho de várias tarefas diárias do indivíduo, tais como subir escadas, caminhar ou até mesmo em situações que poderiam gerar quedas ${ }^{4}$, as chamadas atividades funcionais.

Porém, com o treinamento físico a manutenção do desempenho em atividades diárias parece ser mantida, ou quando há decréscimos, estes são menores, que em idosos sedentários ${ }^{5}$. Desta forma, o treinamento físico se mostra importante, por postergar perdas em relação à potência muscular. Sendo, essa capacidade física dada pela relação força versus velocidade, em que o gesto motor deve ser mais rápido para que se tenha maior potência, o que é necessário para que idosos realizem as atividades de vida diária com melhor desempenho ${ }^{6}$.

Uma forma fácil, validada e amplamente utilizada na literatura científica para mensurar potência de membros inferiores, é através da utilização de testes de saltos propostos por Bosco? . Já que, saltar é uma tarefa motora que envolve uma sequência complexa, multiarticular de movimentos. O máximo desempenho requer coordenação ótima inter-membros do tronco e membros inferiores. Isso representa a transferência de energia eficiente através das articulações envolvidas ${ }^{8}$.

O desempenho do salto é aumentado pelo ciclo alongamento-encurtamento (CAE), um mecanismo que envolve uma contração excêntrica (fase de travagem) antes da concêntrica (fase propulsora). A comparação desses tipos de saltos possibilita o entendimento da contribuição do CAE durante o salto vertical ${ }^{9}$.

Quanto ao ganho na altura devido ao CAE (aumento do pré-alongamento), adultos apresentam no CMJ melhor desempenho, em comparação com o Squat Jump $(\mathrm{SJ})^{10}$. Isso tem mostrado que um trabalho positivo durante o CAE é aumentado com forte intensidade de pré-alongamento, o qual é conseguido pelo aumento de velocidade de descida ${ }^{10,11}$.

Diversos estudos utilizam esses tipos de testes com distintas populações, em especial adultos-jovens ${ }^{12,13}$. No entanto, abordando essa metodologia de avaliação na comparação de idosos com grupos de adultosjovens e com mensuração simultânea da ativação eletromiográfica, a literatura é escassa.

A partir do que foi exposto, o objetivo desse estudo foi verificar se há economia eletromiográfica e/ou aumento nas varáveis dos saltos verticais, ambos em função da utilização do Ciclo AlongamentoEncurtamento e, comparar os achados entre mulheres jovens e idosas, ambas ativas.

\section{MATERIAIS E MÉTODOS}

Inicialmente, para obter a permissão dos participantes da pesquisa, foi encaminhado o Termo de Consentimento Livre e Esclarecido, e respeitada a opção de participar ou não da pesquisa, e aos que participarem foram respeitados o sigilo dos dados coletados.

O presente estudo foi aprovado pelo Comitê de Ética em Pesquisa do Centro Universitário Cenecista de Osório - UNICNEC sob o protocolo no 50191115.7.00005591.

Visando verificar e comparar a utilização do Ciclo Alongamento-Encurtamento na potência de membros inferiores, expressos por variáveis obtidas ao longo dos saltos e o desempenho neuromuscular por meio da eletromiografia em testes de saltos verticais propostos por $\mathrm{Bosco}^{7}$, caracterizando-se por ser uma pesquisa quantitativa.

Para fazerem parte do estudo no grupo "ativo", os indivíduos deveriam apresentar uma prática de atividades físicas somadas aos deslocamentos realizados e demais esportes praticados de no mínimo 150 minutos semanais (30 minutos, cinco dias por semana) com intensidade leve a moderada, seguindo a recomendação tradicional ${ }^{14}$. Independentemente do tipo de grupo (adultas-jovens e idosas), os participantes não poderiam apresentar qualquer tipo de distúrbio vestibular, alteração visual sem correção, diabetes, lesões no sistema musculoesquelético ou dor lombar. 
As avaliações ocorreram no Laboratório de Fisiologia do Exercício do Centro Universitário Cenecista de Osório - UNICNEC, Osório/RS, Brasil. Inicialmente, as participantes preencheram uma ficha de avaliação contendo dados demográficos da amostra (idade, massa corporal, altura, frequência de atividade física e esportiva, presença ou não de lesão, entre outras).

Para mensurar a atividade elétrica muscular foram utilizados eletrodos de superfície na configuração bipolar (AgCL3; modelo Meditrace, da marca 3M), posicionados paralelamente e separados por $20 \mathrm{~mm}$. Os eletrodos foram posicionados longitudinalmente e no sentido das fibras musculares de acordo com as recomendações da SENIAM (Surface EMG for Non-Invasive Assessment of Muscles). Para facilitar a visualização do local do posicionamento dos eletrodos, foi solicitado à participante realizar uma contração dos músculos avaliados, com objetivo de identificação do ventre muscular. Um eletrodo de referência foi posicionado sobre a face anterior da tíbia. Antes da fixação dos eletrodos, foi realizada tricotomia, abrasão e limpeza da pele com algodão e álcool para remover as células mortas e a oleosidade, a fim de reduzir a impedância ${ }^{15}$.

Para aquisição do sinal EMG dos músculos foi utilizado um eletromiógrafo Miotec (Porto Alegre, Brasil), com quatro canais de entrada operando na frequência de $2000 \mathrm{~Hz}$. O sinal captado pelo eletromiógrafo será gravado em um computador no software Miograph (Miotec Equipamentos Biomédicos Ltda, Brasil) para posterior análise.

Para normalização do sinal eletromiográfico foram realizadas contrações isométricas voluntárias máximas (CIVM), nos músculos: bíceps femoral $(\mathrm{BF})$, reto femoral (RF), vasto lateral (VL), e gastrocnêmico medial (GASM), conforme Cardoso et al. ${ }^{16}$.

Os sinais brutos de EMG foram filtrados por um filtro passa-banda de 20 a $500 \mathrm{~Hz}$ de $5^{\text {a }}$ ordem para atenuarem variações nos dados. Antes da realização das tentativas dos saltos, as participantes realizaram um aquecimento na esteira rolante durante cinco minutos (velocidade de $6,5 \mathrm{~km} / \mathrm{h}$ ). Após isso, foram realizadas cinco tentativas dos saltos Squat Jump (SJ) e do Counter Movement Jump (CMJ), para cada participante. Entre cada tentativa, foram dados 30 segundos de descanso. Após a aquisição dos saltos válidos, foi realizada a média dos dois melhores saltos de cada categoria.

$\mathrm{Na}$ análise estatística os dados foram submetidos à estatística descritiva. Foi verificada a normalidade na distribuição dos dados por meio do teste de ShapiroWilk, a homogeneidade por meio do teste de Levene.
Para as comparações na caracterização dos grupos e nas variáveis analisadas, entre os grupos distintos, foi utilizado o teste $\mathrm{t}$ para amostras independentes. Nas comparações dentro do mesmo grupo foi utilizado o teste $\mathrm{t}$ pareado. $\mathrm{O}$ nível de significância para todos os testes foi de $5 \%$.

\section{RESULTADOS}

Os dados da caracterização dos indivíduos são apresentados na Tabela 1 .

$\mathrm{Na}$ Tabela 2, podem-se encontrar as médias e o desvio padrão da atividade eletromiográfica para os quatro músculos avaliados, na realização dos saltos verticais Squat Jump (SJ) e Counter Movement Jump (CMJ) para os grupos idosos (GI) e jovens (GJ), além da probabilidade de significância (p-valor). É possível observar que nenhum dos músculos avaliados no presente estudo apresenta diferenças estatisticamente significativas nas comparações do percentual médio de utilização do CAE. Também, é possível notar que para VL e RF houveram diferenças nas comparações entre os dois tipos de saltos de ambos os grupos, o qual GI necessitou de maior ativação neuromuscular para saltar menos que o GJ.

A Tabela 3 demonstra as médias e os desvios padrão da altura saltada, a potência e a potência normalizada dos saltos verticais, SJ e CMJ, para os grupos idosos (GI) e jovens (GJ), além da probabilidade de significância (p-valor). É possível verificar diferenças estatisticamente significativas na comparação dos grupos na altura saltada, potência e potência normalizada pela massa corporal.

Tabela 1. Médias e desvios padrão de idade e dados antropométricos, além do número de indivíduos em cada grupo.

\begin{tabular}{lcccc}
\hline & \multicolumn{2}{c}{ GI $(\mathbf{n = 1 0})$} & \multicolumn{2}{c}{ GJ $(\mathbf{n = 1 2})$} \\
\cline { 2 - 3 } & Média & $\begin{array}{c}\text { Desvio } \\
\text { padrão }\end{array}$ & Média & $\begin{array}{c}\text { Desvio } \\
\text { padrão }\end{array}$ \\
Idade (anos) & 64,60 & 5,98 & 19,50 & 3,34 \\
Estatura (m) & 1,60 & 0,04 & 1,62 & 0,06 \\
Massa $(\mathrm{kg})$ & 68,02 & 5,12 & 61,16 & 9,77 \\
\hline
\end{tabular}

GI = Grupo idosas; $\mathrm{GJ}=$ Grupo jovens. 
Tabela 2. Médias e desvios padrão da atividade eletromiográfica durante a realização do squat jump (SJ) e counter movement jump (CMJ) para os grupos GI e GJ, além da probabilidade de significância (p-valor).

\begin{tabular}{|c|c|c|c|c|c|c|}
\hline & & \multicolumn{2}{|c|}{ GI $(n=10)$} & \multicolumn{2}{|c|}{ GJ (n=12) } & \multirow[b]{2}{*}{ p-valor } \\
\hline & & Média \pm desvio padrão & $\begin{array}{c}\text { \% médio de } \\
\text { utilização do CAE }\end{array}$ & Média \pm desvio padrão & $\begin{array}{c}\text { \% médio de } \\
\text { utilização do CAE }\end{array}$ & \\
\hline $\begin{array}{l}\text { Vasto lateral } \\
\text { (\% da CVIM) }\end{array}$ & $\begin{array}{l}\text { Squat } \\
\text { CMJ }\end{array}$ & $\begin{array}{l}236,89 \pm 115,66 \\
230,45 \pm 109,10\end{array}$ & $-0,32 \pm 17,17$ & $\begin{array}{c}117,40 \pm 133,09 \\
133,09 \pm 60,71\end{array}$ & $13,32 \pm 16,27$ & $\begin{array}{l}0,071 \\
0,01 *^{\mathrm{Sj}} \\
0,01 *^{\mathrm{Cmj}}\end{array}$ \\
\hline $\begin{array}{l}\text { Reto femoral } \\
\text { (\% da CVIM) }\end{array}$ & $\begin{array}{l}\text { Squat } \\
\text { CMJ }\end{array}$ & $\begin{array}{c}155,42 \pm 49,06 \\
155,45 \pm 61,78\end{array}$ & $0,65 \pm 20,50$ & $\begin{array}{l}106,72 \pm 34,15 \\
108,87 \pm 38,85\end{array}$ & $1,75 \pm 10,35$ & $\begin{array}{l}0,871 \\
0,013 * \mathrm{Sj} \\
0,040 * \mathrm{cmj}\end{array}$ \\
\hline $\begin{array}{l}\text { Bíceps femoral } \\
\text { (\% da CVIM) }\end{array}$ & $\begin{array}{l}\text { Squat } \\
\mathrm{CMJ}\end{array}$ & $\begin{array}{l}64,98 \pm 25,35 \\
64,88 \pm 21,26\end{array}$ & $5,46 \pm 25,64$ & $\begin{array}{l}71,27 \pm 56,23 \\
73,71 \pm 48,26\end{array}$ & $9,10 \pm 21,58$ & $\begin{array}{l}0,722 \\
0,748 * \mathrm{Sj} \\
0,598 * \mathrm{Cmj}\end{array}$ \\
\hline $\begin{array}{l}\text { Gastrocnêmio Medial } \\
\text { (\% da CVIM) }\end{array}$ & $\begin{array}{l}\text { Squat } \\
\text { CMJ }\end{array}$ & $\begin{array}{l}155,56 \pm 44,74 \\
169,08 \pm 37,57\end{array}$ & $7,44 \pm 5,34$ & $\begin{array}{l}122,75 \pm 37,46 \\
127,91 \pm 38,97\end{array}$ & $4,43 \pm 9,63$ & $\begin{array}{l}0,181 \\
0,076^{* \mathrm{Sj}} \\
0,021 * \mathrm{Cmj}\end{array}$ \\
\hline
\end{tabular}

GI = grupo idosas; GJ = grupo jovens; \% da CVIM = percentual da contração voluntária isométrica máxima; CAE = ciclo alongamento-encurtamento.

$*$ sj $=$ probabilidade de significância para Squat; $*$ cmj $=$ probabilidade de significância para CMJ.

Tabela 3. Médias e desvios padrão da altura saltada e potência na realização do squat jump (SJ) e counter movement jump (CMJ) para os grupos GI e GJ, além da probabilidade de significância (p-valor).

\begin{tabular}{|c|c|c|c|c|c|c|}
\hline & & \multicolumn{2}{|c|}{ GI $(n=10)$} & \multicolumn{2}{|c|}{ GJ (n=12) } & \multirow[b]{2}{*}{ p-valor } \\
\hline & & Média \pm desvio padrão & $\begin{array}{c}\text { \% médio de } \\
\text { utilização do CAE }\end{array}$ & Média \pm desvio padrão & $\begin{array}{c}\text { \% médio de } \\
\text { utilização do CAE }\end{array}$ & \\
\hline Altura do salto $(\mathrm{cm})$ & $\begin{array}{l}\text { Squat } \\
\text { CMJ }\end{array}$ & $\begin{array}{c}9,67 \pm 2,80 \\
11,03 \pm 2,76\end{array}$ & $15,69 \pm 11,77$ & $\begin{array}{l}22,93 \pm 5,36 \\
25,44 \pm 5,91\end{array}$ & $11,62 \pm 9,77$ & $\begin{array}{c}0,386 \\
<0,001^{* \mathrm{Sj}} \\
<0,001^{* \mathrm{Cm} j}\end{array}$ \\
\hline Potência (W) & $\begin{array}{l}\text { Squat } \\
\text { CMJ }\end{array}$ & $\begin{array}{l}903,64 \pm 171,96 \\
966,33 \pm 162,82\end{array}$ & $7,44 \pm 5,34$ & $\begin{array}{l}1273,26 \pm 298,91 \\
1344,27 \pm 330,01\end{array}$ & $5,56 \pm 4,60$ & $\begin{array}{l}0,384 \\
0,003 * \mathrm{Sj} \\
0,004 * \mathrm{Cmj}\end{array}$ \\
\hline $\begin{array}{l}\text { Potência normalizada } \\
(\mathrm{W} / \mathrm{kg})\end{array}$ & $\begin{array}{l}\text { Squat } \\
\text { CMJ }\end{array}$ & $\begin{array}{l}13,38 \pm 1,98 \\
14,32 \pm 1,83\end{array}$ & $7,44 \pm 5,34$ & $\begin{array}{l}20,67 \pm 2,51 \\
21,78 \pm 2,56\end{array}$ & $5,56 \pm 4,60$ & $\begin{array}{c}0,384 \\
<0,001 * \mathrm{Sj} \\
<0,001 * \mathrm{Cm} j\end{array}$ \\
\hline
\end{tabular}

GI = grupo idosas; $\mathrm{GJ}=$ grupo jovens; $\mathrm{CAE}=$ ciclo alongamento-encurtamento.

$*$ sj $=$ probabilidade de significância para Squat $; * \mathrm{cmj}=$ probabilidade de significância para CMJ.

\section{DISCUSSÃO}

Percebe-se, na Tabela 2, que todos os músculos em estudo, não apresentaram diferença estatisticamente significativa nas comparações do percentual médio de utilização do CAE. Tal achado, pode ter relação com o método matemático utilizado para cálculo das variáveis somado com a grande variação nos resultados inter-indivíduos na obtenção dos valores de contração isométrica voluntária máxima (CIVM) (em especial ao GI), o que, por sua vez, resultou em valores de desvios padrão muito altos. Sabe-se que para uma correta interpretação dos dados relativos à atividade elétrica dos músculos analisados, a normalização do sinal de EMG é essencial para as comparações entre diferentes dias de coleta, músculos analisados, estudos e, principalmente, entre os indivíduos, numa tentativa de minimizar as diferenças existentes relacionadas à idade, estatura, massa corporal, massa muscular, nível de treinamento e ainda, outros aspectos ${ }^{17}$. Todavia, mesmo que a CIVM seja o melhor e mais usado método de normalização de dados de $\mathrm{EMG}^{18}$, o processo do envelhecimento gera alterações distintas nos indivíduos e essas modificações podem aumentar a variância dos resultados encontrados. Por exemplo, no estudo de Schimidt et al. ${ }^{19}$, os autores observaram diferenças apenas em idosos em comparações de membro dominante e não dominante na força máxima, taxa de produção e força, eficiência neuromuscular, o que não ocorreu com outros dois grupos analisados (crianças e adultos). Essa assimetria apresentada pelos autores em favor do membro dominante é sim um dificultador no processo de normalização e interpretação de dados EMG, pois se para normalizar o sinal se analisa apenas 
a CIVM de um membro (dominante), na execução de tarefas dinâmicas que necessitem os dois membros, como, por exemplo, saltos verticais, podendo haver uma sobrecarga em um dos membros. Além disso, Schimidt et al. ${ }^{19}$ aponta que há uma maior co-ativação de músculos antagonista em idosos que em pessoas jovens.

Porém, mesmo que hajam dificuldades, como as apresentadas acima, os procedimentos de normalização utilizados no presente trabalho são os mais adequados apresentados na literatura ${ }^{18}$, e dados relevantes são encontrados no presente estudo. Entre esses, é possível notar que para VL e RF houve diferença estatisticamente significativa nas comparações entre os dois tipos de saltos de ambos os grupos, o qual GI necessitou de maior ativação neuromuscular para saltar menos que o GJ (Tabela 2).

Como citado acima, sabe-se que devido o processo de envelhecimento a pessoa sofre algumas alterações fisiológicas no sistema neuromuscular, levando a ter decréscimos de força muscular, diminuição de área de secção transversa, causando atrofia muscular, o déficit na inervação das fibras musculares, alterando a porcentagem de tecido muscular contrátil e ocorrendo a chamada sarcopenia ${ }^{20}$.

Nessa perspectiva, o sistema neuromuscular é responsável por inúmeras funções e, com o passar dos anos, algumas são perdidas, como as inervações das fibras musculares do tipo II ${ }^{21}$. Para Rossi e Sader ${ }^{22}$, após os 80 anos de idade, o indivíduo tem redução de $30 \%$ de fibras do tipo II, a redução de força e potência muscular, está relacionada com a atrofia com a referida tipologia de fibras, assim com o processo de envelhecimento perde-se quantidade e qualidade das proteínas contrateis dos músculos ${ }^{21,22}$.

Em um estudo de Porter et al. ${ }^{2}$, os autores mostraram que o VL apresentou uma diminuição de área de secção transversa das fibras do tipo II, durante o processo de envelhecimento, já as fibras de tipo I não apresentaram alterações importantes. Outros estudos apontam que em relação ao número de fibras tanto do tipo I quanto do tipo II diminuem numericamente na mesma proporção ao longo do processo de envelhecimento ${ }^{23}$. Já em um estudo de Lexell et al. ${ }^{24}$ foi realizado uma autópsia no músculo VL com 43 homens com idade entre 15 e 80 anos e encontraram que a atrofia muscular foi diretamente proporcional à idade dos voluntários, assim a atrofia observada se dá em função de uma redução no tamanho de fibras musculares, principalmente do tipo II, e também à perda de fibras musculares sem predominância para os dois tipos de fibra (tipo I e tipo II).

Em relação a essa diminuição de fibras do tipo II, Lexell et al. ${ }^{24}$ mostram que pode estar associado à perda de inervações desse tipo de fibra muscular e posterior reinervação das mesmas por brotamento colateral de axônios de fibras do tipo I, as quais apresentariam características das próprias fibras do tipo I, assim apresentando menor força muscular. Em outro estudo, Pette et al. ${ }^{25}$ mostram que alguns fatores devem ser considerados nessa situação, uma vez que o envelhecimento leva não somente à redução da atividade contrátil, mas também à perda seletiva e remodelamento de unidades motoras (UM) assim, o maior número de fibras do tipo I ocorre devido à atrofia das UM de fibras do tipo II, uma vez que atividades de força muscular não são muito praticadas por essa população ${ }^{25,26}$. Isso reforça a necessidade de realização de exercícios de força em indivíduos idosos, pois a sarcopenia, associada com a maior incidência de fibras lentas nessa população, é a responsável pelo declínio das funções musculares durante o envelhecimento ${ }^{26}$.

Sabe-se ainda que a frequência de disparo das UM remanescentes pode se tornar mais variável ou ainda diminuir com o envelhecimento, fato que levaria à deficiência do controle motor e consequentemente ao comprometimento do desenvolvimento de níveis maiores de força muscular ${ }^{27}$. Então, o fato de que as fibras do tipo II (contração rápida) serem inervadas por fibras do tipo I (contração lenta) o GI necessitaria de maior ativação neuromuscular para realizar o salto. Pelo fato de que o músculo RF apresenta grande número de fibras do tipo II (contração rápida), e por ser um músculo biarticular, tendo como estratégia neural dividir o torque nas duas articulações envolvidas, joelho e quadril, tendo maior ativação neuromuscular para proteger a articulação do joelho e também controlar a direção do movimento ${ }^{28}$.

No caso do músculo BF, o qual não apresentou diferença significativamente estatística, tanto para o uso do CAE quanto na comparação entre os saltos, sendo este um músculo flexor de joelho e extensor do quadril em cadeia fechada, atuando de forma excêntrica na impulsão de ambos os saltos. Já no caso de músculo GASM, apresentou diferença significativamente estatística apenas para o salto CMJ, possivelmente por ser um músculo importante na fase de propulsão do salto vertical, atuando concentricamente na fase de saída do solo realizando flexão plantar gerando maior potência para o voo, e assim obtendo melhor desempenho no salto, assim com o processo de envelhecimento gerando a diminuição de força muscular e a reinervação das fibras musculares do tipo II, o músculo se torna mais fraco necessitando de maior ativação neuromuscular para a realização do salto, em relação ao GJ que por ainda não passarem pelo processo de envelhecimento mantendo assim as fibras musculares do tipo II preservadas, e 
possivelmente sabendo utilizar de melhor forma suas ativações neuromusculares ${ }^{11.29}$.

Por ano, o declínio da força muscular é 3-4\% maior do que o Taxa de diminuição da força muscular ${ }^{30}$. Assim, a força muscular é mais vulnerável ao processo de envelhecimento do que outras variáveis neuromusculares. Portanto, as intervenções para aumentar a força muscular são de fundamental importância para pessoas idosas, a fim de restaurar a mobilidade e desempenho nas atividades de vida diárias ${ }^{31}$.

No estudo de Schimidt et al. ${ }^{19}$ os autores apresentaram que a produção de menor força observada no grupo de idosos foi antecipada, devido aos efeitos de envelhecimento esperados no sistema neuromuscular, a qual tal perda de força foi acompanhada por menor eficiência neuromuscular e a taxa de desenvolvimento de força em comparação com crianças e adultos jovens. A força máxima e as assimetrias de taxa de desenvolvimento de força foram observadas apenas em indivíduos idosos, e sempre a favor do membro preferido. Segundo eles, esses resultados sugerem uma perda neuromuscular geral com significativa assimetria extensora do joelho no grupo idoso, enquanto para crianças e adultos jovens, os índices de assimetria podem não ser relevantes para a avaliação da função neuromuscular, assim torna-se importante na avaliação de indivíduos mais velhos.

A dor e/ou osteoartrite, a mobilidade e o controle do movimento são influenciados pela fraqueza muscular observada nos membros inferiores, especialmente após os 50 anos de idade ${ }^{32}$. A menor taxa de desenvolvimento de força nos idosos pode resultar de mudanças nas tendências morfológicas e mecânicas. A diminuição da rigidez do tendão reduz a transmissão de força do músculo para a inserção óssea e consequentemente diminui a potência ${ }^{33-35}$. Embora as assimetrias de taxa de produção de força e a força, observadas em favor do membro preferido nos idosos possam sugerir alguma adaptação específica entre os membros, o que pode influenciar a transmissão da força durante as contrações isométricas de diferentes maneiras para cada membro e pode causar desequilíbrios específicos durante as atividades da vida diária ${ }^{19}$.

Assim, enquanto os indivíduos idosos apresentavam um padrão de assimetria bem definido na força de extensão do joelho e na taxa de desenvolvimento de força, crianças e adultos jovens não apresentavam assimetria significativa. Uma possível explicação para essa simetria observada nas crianças e adultos jovens é o seu maior envolvimento diário em ações bilaterais, o que contribui para uma habilidade similar entre os membros $^{36}$. Já para os idosos, as assimetrias podem ser explicadas pelo modelo do hemisfério direito e envelhecimento, o qual o hemisfério cerebral direito mostra maior declínio relacionado à idade do que o hemisfério esquerdo assim, uma ampliação global das assimetrias motoras seria esperada com o processo de envelhecimento ${ }^{36}$.

Quanto a menor eficiência neuromuscular observada nos idosos, pode ser o resultado da co-ativação dos músculos antagonistas superiores, como o BF ${ }^{37}$. Além disso, a diminuição do número e da taxa de disparo de unidades motoras ativas no idoso afeta negativamente a capacidade de ativar completamente o músculo, o que também influencia eficiência neuromuscular ${ }^{38}$. MauMoeller et al..$^{39}$, relataram diminuição da atividade muscular voluntária diminuída de idade no torque voluntário isométrico máximo, assim se as mudanças no número e na taxa de disparo das unidades motorizadas ativas se manifestarem de forma específica para cada membro permanecem incertas.

\section{CONCLUSÃO}

O presente estudo permitiu o entendimento de que não houve diferença estatisticamente significativa na utilização do CAE, em ambos os grupos (GI e GJ), pois idosas e jovens tiverem porcentagens similares da utilização do CAE, o que pode vir a ter relação com o método matemático utilizado para cálculo das variáveis somado com a grande variação nos resultados inter-indivíduos na obtenção dos valores de contração isométrica voluntária máxima (CIVM) (em especial ao grupo idoso), o que, por sua vez, resultou em valores de desvios padrão muito altos.

Porém quando se trata sobre a altura saltada e a potência exercida, houve diferenças estatisticamente significativas na comparação dos grupos, o que pode estar relacionado com o processo de envelhecimento e suas consequências ao aparelho locomotor e ao sistema neuromuscular.

\section{REFERÊNCIAS}

1. Pimentel AE, Gentile CL, Tanaka H, et al.. Greater rate of decline in maximal aerobic capacity in endurance-trained than in sedentary men. Journal Applied Physiology. 2003; 94:2406-13.

2. Porter MM, Myint A, Kramer JF, et al. Concentric and eccentric knee extension strength in older and younger men and women. Can J Appl Physiol. 1995;20:429-39.

3. Greenlund LJS, Nair KS. Sarcopenia: consequences, mechanisms, and potential therapies. Mechanisms of Ageing and Development. 2003;124:287-99.

4. Bassey EJ, Fiatarone MA, O'neil EF, et al. Leg extensor power and functional performance in very old men and women. Clin Sci.1992;82:321-7. 
5. Hernandes ESC, Barros JF. Efeitos de um programa de atividade físicas e educacionais para idosos sobre o desempenho em testes de atividades de vida diária. R. bras. Ci. e Mov. 2004;12(2):43-50.

6. Gary RH, John PM, Marcas MB. Effects of Resistance Training on Older Adults. Sports Med. 2004;34(5):329-48.

7. Komi PV, Bosco C. Utilization of stored elastic energy in leg extensor muscles by men and women. Medicine and Science in Sports and Exercise. 1978;10(4):261-5.

8. Luhtanen P, KOMI PV. Segmental contribution to forces in vertical jump. Eur. J. Appl. Physiol. 1978;38(3):181-8.

9. Gerodimos VA, Zafeiridis S. Perkos K. Dipla, et al. The contribution of stretch-shortening cycle and arm-swing to vertical jumping performance in children, adolescents, and adult basketball players. Pediatr. Exerc. Sci. 2008;20(4): 379-89.

10. Bobbert MF, Huijing PA, Van Ingen Schenau GV. Drop jumping. I. The influence of jumping technique on the biomechanics of jumping. Med. Sci. Sports Exerc. 1987; 19(4):332-8.

11. Rosa HB, Oliveira VSL, Santos SL, Prusch SK, Barbosa IM, Lemos LFC. O uso do Ciclo Alongamento-Encurtamento em saltos de atletas de Handebol e praticantes de Musculação do sexo feminino. Revista de Educação Física. 2016;85(3): 274-81.

12. Bosco C, KOMI PV. Influence of aging on the mechanical behavior of leg extensor muscles. Eur. J. Appl. Physiol. Occup. Physiol. 1980;45(2-3):209-19.

13. Temfemo AJ, Hugues K, Chardon SH, et al. Relationship between vertical jumping performance and anthropometric characteristics during growth in boys and girls. Eur. J. Pediatr. 2009; 168(4):457-64.

14. American College of Sports Medicine. ACSM stand position on the appropriate intervention strategies for weight loss and prevention of weight regain for adults. Medicine \& Science in Sports \& Exercise. 2001;33:2145-56.

15. Baroni B, Galvão A, Ritzel C, Diefenthaeler Fernando, et al. Adaptações Neuromusculares de Flexores Dorsais e Plantares a Duas Semanas de Imobilização Após Entorse de Tornozelo. Rev Bras Med Esporte. 2010;16(5):358-62.

16. Cardoso J, Prado A, Iriya $\mathrm{H}$, et al. Atividade eletromiográfica dos músculos do joelho em indivíduos com reconstrução do ligamento cruzado anterior sob diferentes estímulos sensório-motores: relato de casos. Fisioterapia e Pesquisa. 2008;15(1):78-85.

17. Beck TW, Housh TJ, Cramer JT, et al. The Effect of the Estimated Innervation Zone on EMG Amplitude and Center Frequency. Med Sci Sports Exerc. 2007;39:1282-90.

18. Pincivero DM, Coelho AJ, Campy RM, et al. Knee extensor torque and quadriceps femoris EMG during perceptuallyguided isometric contractions. J Electromyogr Kinesiol. 2003;13(2):159-67.

19. Schimidt HL, Machado ÁS, Vaz MA, et al.Isometric muscle force, rate of force development and knee extensor neuromuscular efficiency asymmetries at different age groups. Rev. bras. cineantropom. desempenho hum. 2014; $16 ;(3): 307-15$.

20. Frontera WR, Hughes VA, Fielding RA, Et al. Aging of skeletal muscle: a 12-yr longitudinal study. J. Appl. Physiol. 2000; 88:1321-6.

21. Fleck MPAl. Aplicação da versão em português do instrumento de avaliação de qualidade de vida da OMS
(WHOQOL-100). Revista de Saúde Pública. 1999; 33(2):198-205.

22. Rossi E, Sader CS. Envelhecimento do sistema osteoarticula. In: Freitas EV, Py L, Néri AL, Cançado FAX, Gorzoni ML, Rocha SM. Tratado de Geriatria e Gerontologia. Rio de Janeiro: Guanabara Koogan; 2002. p. 508-14.

23. Rogers MA, Evans WJ. Changes in skeletal muscle with aging: effects of exerci se training. Exere. Sport Sei. Rev. 1993;21:65-102.

24. Lexell, Taylos CC, Strom M. What is the cause of the aging atrophy? J. Neural. Sei. 1988;84:275-94.

25. Pette D, Staron RS. Mammalian skeletal muscle fiber type transitions. Int Rev Cytol. 1997;170:143-223.

26. Wilmore JH, Costil DL. Champaign: Human Kinetics. Physiology of sport and exercise. 2 ${ }^{\mathrm{a}}$ ed. 1999. p. 544-68.

27. Laidlaw DH, Ilodeau M, Enoka RM. Steadiness is reduced and motor unit discharge is more vari-able in older adults. Muscle \& Nerve. 2000;23:600-12.

28. Okano AH, Moraes AC; Bankoff ADP, et al. Respostas eletromiográficas dos músculos vasto lateral, vasto medial e reto femoral durante esforço intermitente anaeróbio em ciclistas. Motriz. 2005;11(1):11-24.

29. Fraga CHW, Bianco R, Gonçalves M. Comparação do sinal EMG e das características da passada em diferentes protocolos de corrida incremental. Rev. bras. Educ. Fís. Esporte. 2012;26(4):599-610.

30. Metter EJ, Conwit R, Tobin J, et al. Age-associated loss of power and strength in the upper extremities in women and men. J Gerontol Series A, Biol Sci Med Sci. 1997;52: B267-B276.

31. Tiggemann CL, Dias CP, Radaelli R, et al. Effect of traditional resistance and power training using rated perceived exertion for enhancement of muscle strength, power, and functional performance. 2016;38-42.

32. Segal NA, Glass NA. Is quadriceps muscle weakness a risk factor for incident or progressive knee osteoarthritis? Phys Sportsmed. 2011;39(4):44-50.

33. Moerch L, Pingel J, Boesen M, et al. The effect of acute exercise on collagen turnover in human tendons: influence of prior immobilization. Euro J Appl Physiol. 2013;(113): 449-55.

34. Lichtwark GA, Barclay CJ. The influence of tendon compliance on muscle power output and efficiency during cyclic contractions. J Exp Biol. 2010;213(5):707-14.

35. Barbosa IM, Prusch SK, Dias EM, et al. Comparação de altura e potência nos saltos verticais entre grupos de mulheres ativas. Rev Bras Presc Fisiol Exerc. 2018; in press.

36. Teixeira LA, De Oliveira DL, Romano RG, et al. Leg preference and interlateral asymmetry of balance stability in soccer players. Res Q Exerc Sport. 2011;82(1):21-7.

37. Ebersole KT, Malek DM. Fatigue and the electromechanical efficiency of the vastus medialis and vastus lateralis muscles. J Athl Train. 2008;43(2):152-6.

38. Klass M, Baudry S, Duchateau J. Age-related decline in rate of torque development is accompanied by lower maximal motor unit discharge frequency during fast contractions. J Appl Physiol. 2008;104(3):739-46.

39. Behrens M, Mau-Moeller A, Bruhn S. Eff ect of Exerciseinduced Muscle Damage on Neuromuscular Function of the Quadriceps Muscle. Int J Sports Med. 2012;33(8): $600-6$. 